\title{
Is informed consent in research sometimes optional?
}

S cientists often complain that the current system for regulating research via research ethics boards in Canada and institutional review boards in the United States is too burdensome, with long, tedious consent forms that don't result in better protection for research participants. Now, leading ethicists writing in the Feb. 20 issue of the New England Journal of Medicine claim that some studies on humans "may not require patient consent at all."

It's a radical idea that goes against the rules. According to the federal Office for Human Research Protections in the US and Canada's Interagency Advisory Panel on Research Ethics, people have the right to choose whether to participate in a research project after hearing about it. But Nancy Kass, a professor at Johns Hopkins University in Baltimore, Maryland, and one of the authors of the New England Journal of Medicine article, told CMAJ that the rules are applied too broadly. She compared investigators conducting studies of approved, standard treatments, where the risks to patients are very small, to doctors taking a patient's blood pressure. Doctors don't specifically inform patients about why they're checking blood pressure or ask permission to do it, she said; they make "an assumption based on experience, that patients don't mind, that they understand it's not harmful and it's in their best interest." Certain research to improve clinical care "fits into that same context," she said.

In the US, the legislation that created Obamacare made billions of dollars available for scientists to compare standard treatments to see which are more effective. Some projects were no doubt held up as a result of ethics review. Kass and coauthors write that a novel ethics framework is needed because current practices make research to prevent medical errors or improve medical management "unduly burdensome to conduct."

In the same issue of the New England Journal of Medicine, ethicists at the National Institutes of Health Clinical Center in Bethesda, Md., argue against a no-consent model, writing that it would lead physicians to conceal studies from

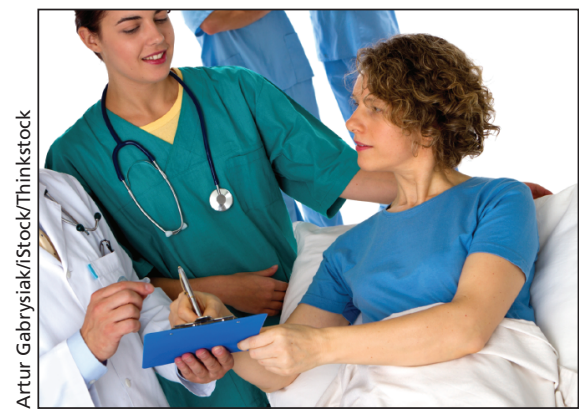

Research that is not harmful should not require consent, argue some US ethicists.

patients, which "could erode trust." A more workable solution may be what Kass' group refers to as streamlined consent - where patients hear about a study and have the chance to opt out, but do not sign consent forms.

Experts on both sides of the border agree that the hurdles blocking low-risk research are a problem. Ann Heesters, a bioethicist with the University of Toronto's Joint Centre for Bioethics in Ontario, said the current review process is "too inflexible" to allow for "research that doesn't fit the mold." The result is that scientists sometimes sidestep review by describing low-risk projects in ways that do not count as research.

The harshest critics of the current ethics board system in the US and Canada claim that it is literally costing lives because it makes doing life-saving research so unwieldy. Kass points to advances in treating pediatric cancer and leukemia, which came after the overwhelming majority of children with those diseases obtained treatment via research protocols. But it's an unusual example. "Essentially," she said, "when no one was paying attention, it turned out that we are systematically harming patients' interests by making it really difficult to do certain kinds of research." - Miriam Shuchman, Toronto, Ont.

CMAJ 2014. DOI:10.1503/cmaj.109-4753

\section{More News online}

To read more $C M A J$ news articles, visit cmaj.ca/site/home/news.xhtml 